\title{
Prenatal diagnosis and molecular cytogenetic characterization of two hereditary chromosomal duplications with favorable
}

\section{outcomes}

\author{
Y.J. Wu ${ }^{1, \dagger}$, C.J. Yu ${ }^{2, \dagger}$, W.H. Tian ${ }^{3, \dagger}, \mathbf{Z} . \mathbf{X u}^{4, *}$ \\ ${ }^{1}$ Department of Obstetrics, Huanggang Central Hospital, Huanggang, Hubei (P.R. China) \\ ${ }^{2}$ Department of Prenatal Diagnosis Center, Maternal and Child Health Hospital of Hubei Province, Wuhan, Hubei (P.R. China) \\ ${ }^{3}$ Department of Reproductive Medicine Centre, Hubei Enshi Tujia and Miao Autonomous Prefecture Central Hospital, \\ Enshi, Hubei (P.R. China) \\ ${ }^{4}$ Department of Gynecology, Maternal and Child Health Hospital of Hubei Province, Wuhan, Hubei (P.R. China)
}

\begin{abstract}
Summary
The objective of this study was to report two cases of hereditary chromosomal duplications with favorable outcomes. In both cases, conventional karyotyping showed a normal karyotype. However, chromosomal microarray analysis on uncultured amniocytes detected a $3.2 \mathrm{Mb}$ duplication in the region of $\operatorname{arr}[\mathrm{hg} 19]$ 13q12.11q12.12(22,073,046-25,230,759) $\times 3$ in case 1 and a $3.1 \mathrm{Mb}$ duplication in the region of $\operatorname{arr}[\mathrm{hg} 19] 6 \mathrm{q} 12(65,423,142-68,550,465) \times 3$ in case 2 . In both cases, the chromosomal duplication was inherited from a mother who has no symptoms. Both cases resulted in phenotypically normal babies.
\end{abstract}

Key words: Conventional karyotyping; Chromosomal microarray; Microduplication; Prenatal diagnosis; Copy number variation (CNV).

\section{Introduction}

The risk of chromosomal abnormality increases with maternal age [1]. The advanced maternal age has been viewed as a sole indication for genetic amniocentesis [2]. Amniocentesis is a procedure that takes out a small sample of the amniotic fluid for testing. Conventional karyotyping provides an overview of the entire genome. It is recommended when a common aneuploidy such as trisomy $21,18,13$ or monosomy $\mathrm{X}$ is suspected based on prenatal ultrasound examination [3]. However, conventional karyotyping can't detect aberrations that are less than $5 \mathrm{Mb}$ in length [4].

Chromosomal microarray is a new technology to detect chromosome abnormalities that are less than $5 \mathrm{Mb}$ in length [5]. With chromosomal microarray, DNA from a fetal sample such as amniocentesis is hybridized to an array platform consisting of DNA probes on a solid surface. It allows detecting aberrations down to $50 \mathrm{~kb}$ [5]. Because chromosomal microarray does not require cell culture, it offers the opportunity for a fast turnaround time [5]. However, chromosol microarray cannot detect balanced chromosomal rearrangements such as translocations and inversions, which can be detected by conventional karyotyping [5]. Therefore, combining these two methods may increase the diagnostic accuracy for chromosomal abnormalities. In this paper, we presented two cases of hereditary chromosomal duplications with favorable outcomes.

\section{Case Presentation}

Case 1

A 39-year-old, gravida 3, para 1, woman underwent amniocentesis at 20 weeks of gestation because of advanced maternal age. Her husband was 42 years old. Their first child was healthy. There was no family history of birth defects. Conventional karyotyping revealed a normal karyotype of 46, XY. However, chromosomal microarray analysis on uncultured amniocytes using the Affymetrix (Affymetrix, Inc., Santa Clara, CA) SNP 6.0 platform revealed a $3.2 \mathrm{Mb}$ VUS (variant uncertain significance) chromosomal duplication $\operatorname{arr}[\mathrm{hg} 19] 13 \mathrm{q} 12.11 \mathrm{q} 12.12(22,073,046-25,230,759) \times 3$ (Figure 1). Conventional karyotyping using peripheral blood showed a normal karyotype in both parents and their first child. In contrast, chromosomal microarray analysis identified the same duplication in the mother. The father and their first child were normal by chromosomal microarray analysis.

Ultrasound examination showed no dysmorphisms and intrauterine growth restrictions (IUGRs). The pregnancy carried to 40 weeks of gestation and a healthy baby was delivered vaginally. The infant was phenotypically normal and had normal growth and psychomotor development at 6 months old. 


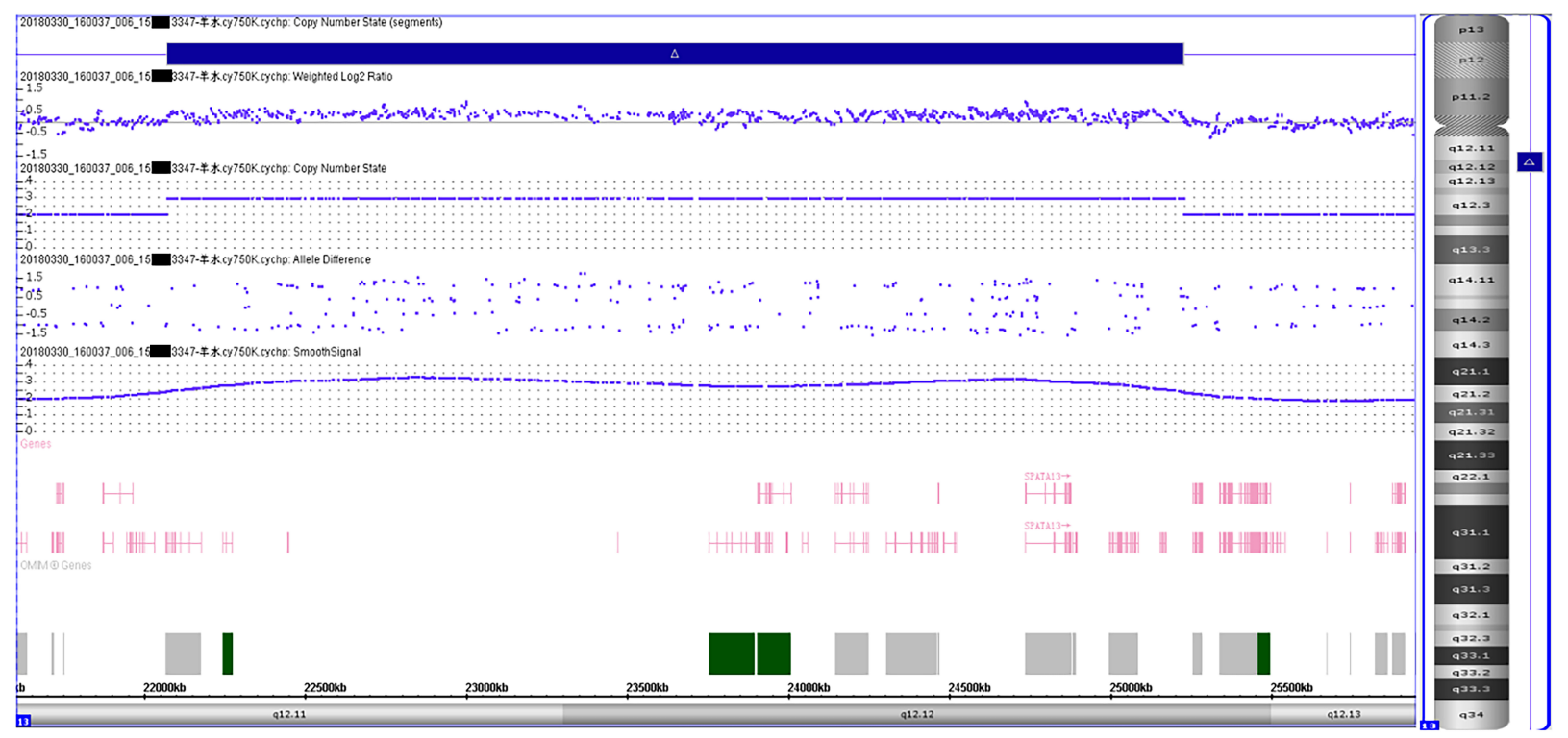

Figure 1. - Chromosomal microarray analysis on uncultured amniocytes using the Affymetrix SNP 6.0 platform revealed a $3.2 \mathrm{Mb}$ duplication on chromosome $\operatorname{arr}[$ hg19] 13q12.11q12.12(22,073,046-25,230,759) $\times 3$.

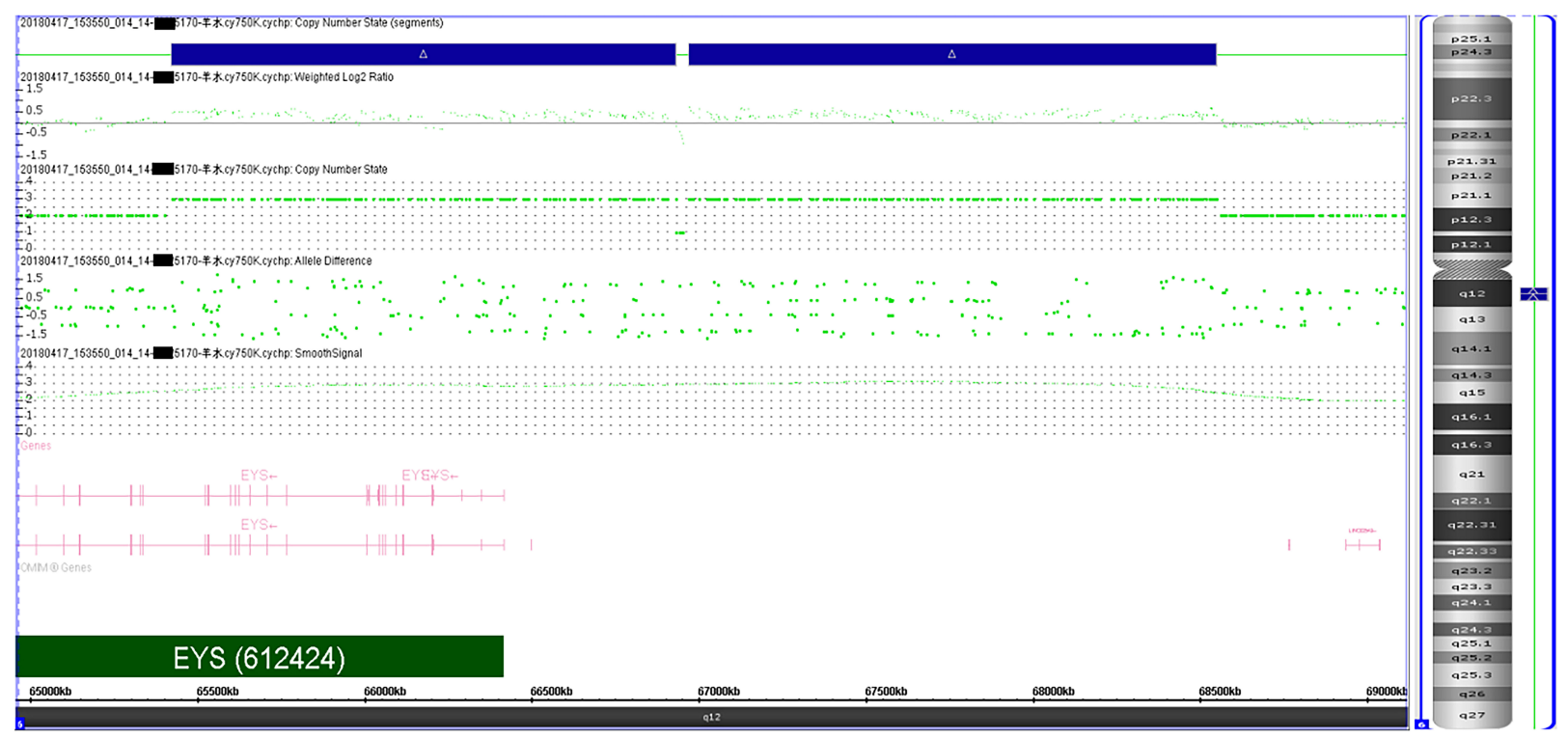

Figure 2. - Chromosomal microarray analysis on uncultured amniocytes using the Affymetrix SNP 6.0 platform revealed a $3.1 \mathrm{Mb}$ duplication on chromosome $\operatorname{arr}[\mathrm{hg} 19] 6 \mathrm{q} 12(65,423,142-68,550,465) \times 3$.

Case 2

A 34-year-old, gravida 2, para 0, woman underwent amniocentesis at 20 weeks of gestation. Her husband was 34 years old. Conventional karyotyping revealed a normal karyotype of 46, XX. However, chromosomal microarray analysis on uncultured amniocytes using the Affymetrix (Affymetrix, Inc., Santa Clara, CA) SNP 6.0 platform revealed a $3.1 \mathrm{Mb}$ chromosomal duplication in the region of $6 q 12(65,423,142-68,550,465) \times 3$ (Figure 2). Conventional karyotyping using peripheral blood revealed a normal karyotype in both parents. However, chromosomal microarray analysis identified the same duplication in the mother. The father was normal by the chromosomal microarray analysis.

Ultrasound examination showed no dysmorphisms and intrauterine growth restrictions (IUGRs). The pregnancy carried to 40 weeks of gestation and a healthy baby was delivered vaginally. The infant was phenotypically normal and had normal growth and psychomotor development at 6 months old.

\section{Discussion}

Women that are above 35 years of age at delivery are usually referred for genetic counseling or prenatal diagnosis such as amniocentesis. In this study, we have demonstrated 
that combining the conventional karyotyping and chromosomal microarray could increase the detection of genetic abnormalities in these women.

In case 1 , we detect a $3.2 \mathrm{Mb}$ VUS chromosomal duplication in the region of $\operatorname{arr}[\mathrm{hg} 19]$ $13 q 12.11 q 12.12(22,073,046-25,230,759)$. In an attempt to further delineate clinical phenotypes of this duplication, we performed a review of the literature and the Decipher and ClinGen database. We identified six cases of isolated duplications that are partially overlapped with our case. All these six cases are associated with a normal phenotype. This region contains 19 genes: MICU2, FGF9, LINC00424, BASP1P1, SGCG, SACS, SACS-AS1, LINC00327, TNFRSF19, MIPEP, C1QTNF9BAS1, C1QTNF9B, ANKRD20A19P, SPATA13, MIR2276, SPATA13-AS1, C1QTNF9, PARP4, TPTE2P6. Among these 19 genes, MICU2 is a $\mathrm{Ca}^{2+}$ sensor protein of mitochondrial uniporter (MCU) mediating mitochondrial $\mathrm{Ca}^{2+}$ uptake [6]. Diseases associated with MICU2 include Loeys-Dietz Syndrome, which is characterized by a broad spectrum of craniofacial, vascular and skeletal manifestations [7]. Mutations in the $S G C G$ gene is associated with an autosomal recessive disease called Limb-girdle muscular dystrophy type $2 \mathrm{C}$ (LGMD2C). The human FGF9 gene encodes the glia-activating factor, which is a member of the fibroblast growth factor family. Diseases associated with FGF9 include Multiple Synostoses Syndrome 3 and Multiple Synostoses Syndrome [8]. We didn't detect any symptoms of these potential diseases in the mother. Therefore, we suspected that this micro duplication might be a nonpathogenic $\mathrm{CNV}$.

In case 2, we detected a $3.1 \mathrm{Mb}$ VUS chromosomal duplication in the region of $\operatorname{arr}[\mathrm{hg} 19] 6 \mathrm{q} 12(65,423,142-$ $68,550,465)$. We found several cases with isolated duplications that are partially overlapped with our case in the Decipher and ClinGen databases. All these cases are associated with normal phenotypes. This region contains three genes: EYS, LOC441155, and SLC25A51P1. It has been previously reported that $6 \mathrm{q} 12$ variants are associate with postnatal exhales nitric oxide levels and respiratory symptoms [9]. However, there are no evidence showing that duplication of this region is associated with any disease. we didn't detect any of the potential reparatory symptoms in the mother.

In both cases, conventional karyotyping showed a normal phenotype. No abnormalities were detected on ultrasound. However, chromosomal microarray analysis detected a novel VUS chromosomal duplication. They are likely to be benign based on current observations. However, the long-term effects of these variants are unclear.

\section{Conclusions}

Hereditary chromosomal duplications may be associated with favorable outcomes. Combining conventional karyotyping, chromosomal microarray analysis and ultrasound can provide a more accurate risk assessment for pregnant women with advanced maternal age.

\section{Abbreviations}

$\mathrm{CNV}$, copy number variation; VUS, variant uncertain significance.

\section{Ethics Approval and Consent to Participate}

This study was approved by the Research Ethics Committee of Maternal and Child Health Hospital of Hubei Province (Ethics approval number: 2018-023-55). All participants were informed of the purpose of this study, and written consent was obtained from each participant prior to sample collection.

\section{Acknowledgments}

We would like to thank Dr. Dan Yu (QB3, UCSF) for the help in revising the manuscript.

\section{Conflict of Interest}

The authors declare no conflict of interest.

Submitted: September 05, 2019

Accepted: April 29, 2020

Published: October 15, 2020

\section{References}

[1] Wieacker P., Steinhard J.: "The prenatal diagnosis of genetic diseases". Dtsch. Arztebl. Int., 2010, 107, 857-862.

[2] Bornstein E., Lenchner E., Donnenfeld A., Barnhard Y., Seubert D. Divon M.Y.: "Advanced maternal age as a sole indication for genetic amniocentesis; risk-benefit analysis based on a large database reflecting the current common practice". J. Perinat. Med., 2009, 37, 99-102.

[3] Deans Z.C., Allen S., Jenkins L., Khawaja F., Hastings R.J., Mann K., et al.: "Recommended practice for laboratory reporting of noninvasive prenatal testing of trisomies 13,18 and 21: a consensus opinion". Prenat. Diagn., 2017, 37, 699-704.

[4] Gonzales P.R., Carroll A.J., Korf B.R.: "Overview of clinical cytogenetics”. Curr. Protoc. Hum. Genet., 2016, 89, 8.1.1-8.1.13.

[5] Levy B., Wapner R.: "Prenatal diagnosis by chromosomal microarray analysis". Fertil. Steril., 2018, 109, 201-212.

[6] Pathak T., Trebak M.: "Mitochondrial $\mathrm{Ca}(2+)$ signaling". Pharmacol. Ther, 2018, 192, 112-123.

[7] Petrungaro, C., Zimmermann, K.M., Küttner, V., Fischer, M., Dengjel, J., Bogeski, I., et al.: "The $\mathrm{Ca}(2+)$-dependent release of the Mia40-induced MICU1-MICU2 dimer from MCU regulates mitochondrial $\mathrm{Ca}(2+)$ uptake". Cell Metab., 2015, 22, 721-733.

[8] Wu X.L., Gu M.M., Huang L., Liu X.S., Zhang H.X., Ding X.Y., et al.: "Multiple synostoses syndrome is due to a missense mutation in exon 2 of FGF9 gene". Am. J. Hum. Genet., 2009, 85, 53-63.

[9] Fuchs O., Gorlanova O., Latzin P., Schmidt A., Schieck M. Toncheva A.A., et al.: "6q12 and 11 p14 variants are associated with postnatal exhaled nitric oxide levels and respiratory symptoms". $J$. Allergy Clin. Immunol., 2017, 140, 1015-1023.

Corresponding Author:

ZHEN XU, M.D.

Department of Gynecology, Maternal and Child Health Hospital of Hubei Province, Wuhan, Hubei (P.R. China)

e-mail: xuzhenzhen2020@163.com 